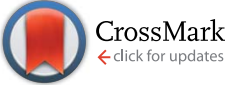

Cite this: RSC Adv., 2016, 6, 87767

\title{
Polyhydroxylated few layer graphene for the preparation of flexible conductive carbon paper
}

\begin{abstract}
Vincenzina Barbera, ${ }^{a}$ Alessandro Porta, ${ }^{a}$ Luigi Brambilla, ${ }^{a}$ Silvia Guerra, ${ }^{a}$ Andrea Serafini, ${ }^{a}$ Antonio Marco Valerio, ${ }^{a}$ Alessandra Vitale ${ }^{b}$ and Maurizio Galimberti ${ }^{\star a}$

Electrically conductive flexible carbon papers were prepared, based on hydroxyl functionalized few layer graphene $(\mathrm{G}-\mathrm{OH})$. G-OH was obtained from the reaction of $\mathrm{KOH}$ with nanosized graphite with a very high surface area (HSAG), greater than $300 \mathrm{~m}^{2} \mathrm{~g}^{-1}$, and with high shape anisotropy, with the help of mechanical and thermal energy. Wide angle X-ray and Raman analyses showed that the core of $\mathrm{G}-\mathrm{OH}$ had the structure of infinite and ideal graphene layers and that the interlayer distance between the few stacked graphene layers was the same as in pristine HSAG. Hydroxyl groups were thus essentially located in peripheral positions. High resolution transmission electron microscopy revealed the presence of graphene aggregates made by a number of layers as low as 6 . Stable water suspensions were obtained, with concentrations up to $4 \mathrm{mg} \mathrm{mL}^{-1}$. Mild centrifugation of such suspensions had interesting efficiency as a method for producing few layer graphene: about $35 \%$ by mass of pristine HSAG was isolated as few layer graphene from supernatant suspensions. Flexible and electrically conductive carbon papers were prepared by coating a paper support with a $\mathrm{G}-\mathrm{OH}$ water suspension. This work demonstrates that carbon papers can be prepared without adopting the traditional oxidation-reduction procedure, avoiding harsh reaction conditions, dangerous and toxic reagents, solvents and catalysts.
\end{abstract}

Received 27th July 2016

Accepted 4th August 2016

DOI: $10.1039 /$ c6ra19078b

www.rsc.org/advances promote the dispersion of the modified graphitic layers in water and other polar solvents, allowing the preparation of coating layers. This is reported in the literature as a key step for the preparation of graphene papers, one of the investigated applications of graphene. However, this approach to the preparation of graphene papers is affected by some main drawbacks. Oxidation, typically performed through Staudenmaier ${ }^{13}$ or Hummers ${ }^{14}$ methods, requires explosive oxidation agents, strong acids, harsh reaction conditions and long reaction times. Moreover, it is widely acknowledged that oxidation leads to extensive disruption of $\mathrm{sp}^{2}$ hybridization of graphene layers, with loss of properties. The reduction step is thus ineluctable, to restore the graphene layers and in turns, to allow the preparation of conductive graphene papers. However, chemicals for the reduction could be toxic, hazardous and expensive, such as, for instance, those based on hydrazine. ${ }^{20-22}$ Moreover, high temperature is needed for thermal reduction. ${ }^{23,24}$ Other reduction methods have been recently reported, for the obtainment of graphene papers $\mathrm{s}^{25-29}$ Thermal treatments at $700{ }^{\circ} \mathrm{C}$ have been applied to graphene oxide paper, under argon or hydrogen atmosphere. ${ }^{25}$ Supercritical ethanol was used. ${ }^{26} \mathrm{Ar}^{+}$ion irradiation was performed on GO paper obtained by vacuum drying of water dispersion on stainless steel plate. ${ }^{27}$ Reduced grapheneoxide ( $\mathrm{r}-\mathrm{GO})$ papers, prepared by vacuum filtration, were treated with $\mathrm{Ar} / \mathrm{H}_{2}$ atmosphere..$^{28} \mathrm{GO}$ film on a PET substrate (PET) was reduced with a magnetron sputtered Sn metal layer. ${ }^{29}$ However, all the approaches documented in the literature for the
${ }^{a}$ Politecnico di Milano, Department of Chemistry, Materials and Chemical Engineering “G. Natta”, Via Mancinelli 7, 20131 Milano, Italy. E-mail: maurizio.galimberti@ polimi.it

${ }^{b}$ Politecnico di Torino, Department of Applied Science and Technology DISAT, Corso Duca degli Abruzzi 24, 10129 Torino, Italy

$\dagger$ Electronic supplementary information (ESI) available. See DOI: 10.1039/c6ra19078b 
preparation of graphene papers are based on two steps: preparation of GO and successive reduction.

In the light of this situation, it appears worthwhile to make research for an efficient, simple method for the preparation of graphene and/or few layers graphene, suitable for the preparation of flexible and conductive carbon papers. Ideally, such method should be based on a single step, in line with the basic principles of green chemistry. The introduction of functional groups on graphene layers, preferentially oxygenated functional groups, would be highly desirable, as well as the preservation of the $\mathrm{sp}^{2}$ nature of carbon atoms.

This work had these objectives. Oxidation of a nanosized high surface area graphite (HSAG) was pursued through the reaction with $\mathrm{KOH}$, with the aim to selectively introduce hydroxyl groups on edges of graphene layers, leaving substantially unaltered the bulk crystalline order and thus the aromatic nature of the layers. Further aim was to promote exfoliation of HSAG, achieving the preparation of few layers graphene. Elimination of hydroxyl groups was not pursued. On the contrary, functionalized graphene layers were used as macro-substrates for further functionalization reactions. The one with isocyanate to form urethanes is reported as an example. Further objective was the obtainment of few layers graphene suspensions in ecofriendly solvents such as water and the preparation of flexible and electrically conductive carbon papers by deposing G$\mathrm{OH}$ suspensions on different substrates.

To pursue such objectives, strategic was the selection of the nanosized graphite. HSAG had a very high surface area, higher than $300 \mathrm{~m}^{2} \mathrm{~g}^{-1}$, and a high shape anisotropy, defined as the ratio between the crystallites size in directions perpendicular and orthogonal to the layers. ${ }^{30}$ In particular, HSAG had a low number of stacked layers, about three tenths. Reaction of HSAG with $\mathrm{KOH}$ was performed in a single step, with the help of either mechanical or thermal energy. Few layers graphenes with $\mathrm{OH}$ functional groups $(\mathrm{G}-\mathrm{OH})$ were characterized by means of elemental and thermogravimetric analysis (TGA), high resolution X-ray photoelectron spectroscopy (XPS), infrared (IR) and Raman spectroscopy, wide angle X-ray diffraction (WAXD), high-resolution transmission electron microscopy (HRTEM) and cyclic voltammetry. Dispersions in water of G-OH were prepared and investigated through dynamic light scattering (DLS) and UV spectroscopy. Mild centrifugation of such dispersions and recovery from supernatant was studied as a method to obtain few layer graphene. Thin films were prepared on paper substrate starting from a water suspensions of G-OH. Flexibility and electrical conductivity of the obtained carbon papers were investigated.

\section{Experimental}

\subsection{Materials}

Reagents and solvents commercially available were purchased and used without further purification: potassium hydroxide pellets (Carlo Erba Reagenti), acetone (Aldrich), carboxymethylcellulose sodium (Sigma-Aldrich), high surface area graphite (HSAG) with Synthetic Graphite $8427 \circledR$ as trademark was purchased from Asbury Graphite Mills Inc., with a minimum carbon mass $\%$ of 99.5 , a surface area of $330 \mathrm{~m}^{2} \mathrm{~g}^{-1}$, the number of stacked layers in the orthogonal direction to graphitic planes is about $35 .{ }^{30} \mathrm{GO}$ was prepared by Staudenmaier's method, ${ }^{2}$ as reported in an our previous work. ${ }^{30}$

\subsection{Structural characterization of graphitic starting material}

The selection of pristine graphite was performed after WAXD characterization of many disordered graphitic samples. HSAG was selected as it was shown to have a turbostratic structure, with high crystalline order inside the structural layers and a relatively low number of stacked layers. ${ }^{30}$ In the technical data sheet, the carbon content and the surface area are reported to be at least $99 \%$ by mass and $330 \mathrm{~m}^{2} \mathrm{~g}^{-1}$, respectively. Chemical composition determined from elemental analysis was, as mass\%: carbon 99.5, hydrogen 0.4 , nitrogen 0.1 , oxygen 0.0 . BET surface area was determined by applying ASTM D6556 method and was found to be $330.3 \mathrm{~m}^{2} \mathrm{~g}^{-1}$.

\subsection{Synthesis of hydroxy-graphite (G-OH)}

2.3.1. Thermal treatment (G-OH-T). In a $100 \mathrm{~mL}$ round bottom flask equipped with magnetic stirrer were poured in sequence pristine graphite $(2.81 \mathrm{~g}, 39 \mathrm{mmol})$, $\mathrm{KOH}$ powder (2.19 $\mathrm{g}, 39 \mathrm{mmol})$ and $\mathrm{H}_{2} \mathrm{O}(22 \mathrm{~mL})$. The mixture was left to stir at 70 ${ }^{\circ} \mathrm{C}$ for 4 hours. After this period, the resulting mixture was first kept to room temperature and removed from the flask using deionized water $(5 \mathrm{~mL})$. The so obtained suspension was kept in a Büchner funnel with a sintered glass disc and was purified from the excess of $\mathrm{KOH}$ washing repeatedly with distilled water $(6 \times 100 \mathrm{~mL})$ under vacuum. The obtained solid was put in an oven to remove excess water. It were obtained $2.60 \mathrm{~g}$ of black powder. ATR-FTIR $\nu_{\max } 3408$ (O-H stretch, broad), 1347 (in plane $\mathrm{O}-\mathrm{H}$ bend), 1120 (C-O stretch), 989 ( $\mathrm{C}=\mathrm{C}$ bend), 856 (out of plane, $=\mathrm{C}-\mathrm{H}$ bend, monosubst) $\mathrm{cm}^{-1}$.

2.3.2. Mechanical treatment (G-OH-M) - method A. The synthesis of hydroxy-graphite (G-OH) was performed using a planetary ball mill S100 from Retsch, having the grinding jar moving in a horizontal plane, with a volume of $0.3 \mathrm{~L}$. The grinding jar was loaded with 6 ceramic balls having a diameter of $20 \mathrm{~mm}$. Graphite ( $1 \mathrm{~g}, 14 \mathrm{mmol}$ ), $\mathrm{KOH}$ powder $(20 \mathrm{~g}, 356$ $\mathrm{mmol})$ and $\mathrm{H}_{2} \mathrm{O}(6.5 \mathrm{~mL})$ were put into the jar, that was allowed to rotate at $300 \mathrm{rpm}$, at room temperature, for 10 hours. After this time, the mixture was placed in a Büchner funnel with a sintered glass disc and repeatedly washed with distilled water $(6 \times 100 \mathrm{~mL})$ under vacuum. Finally, the obtained solid was put in an oven to remove excess water. It were obtained $0.65 \mathrm{~g}$ of black powder. ATR-FTIR $\nu_{\max } 3390$ (O-H stretch, broad), 1396 (in plane $\mathrm{O}-\mathrm{H}$ bend), 1120 (C-O stretch), 983 ( $\mathrm{C}=\mathrm{C}$ bend), 850 (out of plane, $=\mathrm{C}-\mathrm{H}$ bend, monosubst) $\mathrm{cm}^{-1}$.

2.3.3. Mechanical treatment (G-OH-M) - method B. The synthesis of hydroxy-graphite (G-OH) was performed using a planetary ball mill S100 from Retsch, having the grinding jar moving in a horizontal plane, with a volume of $0.3 \mathrm{~L}$. The grinding jar was loaded with 6 ceramic balls having a diameter of $20 \mathrm{~mm}$. Graphite (13 g, $0.18 \mathrm{~mol}$ ), $\mathrm{KOH}$ powder (100 g, 1.78 $\mathrm{mol}$ ) were put into the jar that was allowed to rotate at $300 \mathrm{rpm}$, at room temperature, for 15 hours. After this time, the mixture 
was placed in a Büchner funnel with a sintered glass disc. It was repeatedly washed with distilled water $(6 \times 100 \mathrm{~mL})$ under vacuum. The resulting solid was put in an oven to remove water. It were obtained $12.2 \mathrm{~g}$ of black powder. ATR-FTIR $\nu_{\max } 3390$ (O-H stretch, broad), 1396 (in plane O-H bend), 1120 (C-O stretch), 983 ( $\mathrm{C}=\mathrm{C}$ bend), 850 (out of plane, $=\mathrm{C}-\mathrm{H}$ bend, monosubst) $\mathrm{cm}^{-1}$.

2.3.4. Thermal and mechanical treatment (G-OH-TM). In a $250 \mathrm{~mL}$ round bottom flask equipped with magnetic stirrer were poured pristine graphite $(15 \mathrm{~g}, 208.3 \mathrm{mmol}), \mathrm{KOH}$ powder (11.69 g, $208.3 \mathrm{mmol}$ ) and $\mathrm{H}_{2} \mathrm{O}(117 \mathrm{~mL})$. The mixture was left to stir at $70{ }^{\circ} \mathrm{C}$ for 4 hours. After this period the resulting mixture was first kept to room temperature and removed from the flask using deionized water ( $5 \mathrm{~mL}$ ). The suspension was dried under vacuum and the resulting powder was placed in a planetary ball mill S100 from Retsch, having the grinding jar moving in a horizontal plane, with a volume of $0.3 \mathrm{~L}$. The grinding jar was loaded with 6 ceramic balls having a diameter of $20 \mathrm{~mm}$. The jar was allowed to rotate at $300 \mathrm{rpm}$, at room temperature, for 5 hours. After this time the powder was moved in four Falcon where was added deionized water and centrifuged at $9000 \mathrm{rpm}$ for 10 minutes. The deionized water was changed three times to completely remove all impurities. The obtained solid was put in an oven to remove water traces. $13.7 \mathrm{~g}$ of black powder were obtained. ATR-FTIR $\nu_{\max } 3394$ (O-H stretch, broad), 1390 (in plane $\mathrm{O}-\mathrm{H}$ bend), 1147 (C-O stretch), 995 (C=C bend), 721 (out of plane, $=\mathrm{C}-\mathrm{H}$ bend, monosubst) $\mathrm{cm}^{-1}$.

\subsection{Characterization of G-OH adducts}

2.4.1. Thermogravimetric analysis. TGA tests under flowing $\mathrm{N}_{2}$ (60 mL $\mathrm{min}^{-1}$ ) were performed with a Mettler TGA SDTA/851 instrument according to the standard method ISO9924-1. Samples $(10 \mathrm{mg})$ were heated from 30 to $300{ }^{\circ} \mathrm{C}$ at $10^{\circ} \mathrm{C} \mathrm{min}^{-1}$, kept at $300{ }^{\circ} \mathrm{C}$ for $10 \mathrm{~min}$, and then heated up to $550{ }^{\circ} \mathrm{C}$ at $20^{\circ} \mathrm{C}$ $\mathrm{min}^{-1}$. After being maintained at $550{ }^{\circ} \mathrm{C}$ for $15 \mathrm{~min}$, they were further heated up to $900{ }^{\circ} \mathrm{C}$ and kept at $900{ }^{\circ} \mathrm{C}$ for 30 min under flowing air $\left(60 \mathrm{~mL} \min ^{-1}\right)$.

2.4.2. Elemental analysis. Elemental analysis was performed with a Thermo FlashEA 1112 Series CHNS-O analyzer, after pretreating samples in an oven at $100{ }^{\circ} \mathrm{C}$ for $12 \mathrm{~h}$.

2.4.3. FT-IR. The IR spectra were recorded in transmission mode (128 scan and $4 \mathrm{~cm}^{-1}$ resolution) in a diamond anvil cell (DAC) using a ThermoElectron FT-IR Continu $\mu \mathrm{m}$ IR microscope.

2.4.4. Raman spectroscopy. Raman spectra of powder samples deposited on a glass slide were taken with a Horiba Jobin Yvon Labram HR800 dispersive Raman spectrometer equipped with Olympus BX41 microscope and a $50 \times$ objective. The excitation line at $632.8 \mathrm{~nm}$ of a He/Ne laser was kept at 0.5 $\mathrm{mW}$ in order to prevent samples degradation. The spectra were obtained as the average of four acquisitions (scan time: 30 seconds for each acquisition) with a spectral resolution of $2 \mathrm{~cm}^{-1}$.

2.4.5. Wide angle X-ray diffraction. Wide-angle X-ray diffraction (WAXD) patterns were obtained in reflection, with an automatic Bruker D8 Advance diffractometer, with nickel filtered $\mathrm{Cu}-\mathrm{K} \alpha$ radiation. Patterns were recorded in $10-100^{\circ}$ as the $2 \theta$ range, being $2 \theta$ the peak diffraction angle. Distance between crystallographic planes was calculated from the Bragg law. The $D_{h k l}$ correlation length, in the direction perpendicular to the $h k l$ crystal graphitic planes, was determined applying the Scherrer equation.

$$
D_{h k l}=K \lambda /\left(\beta_{h k l} \cos \theta_{h k l}\right)
$$

where: $K$ is the Scherrer constant, $\lambda$ is the wavelength of the irradiating beam (1.5419 $\AA, \mathrm{Cu}-\mathrm{K} \alpha), \beta_{h k l}$ is the width at half height, and $\theta_{h k l}$ is the diffraction angle. The instrumental broadening, $b$, was determined by obtaining a WAXD pattern of a standard silicon powder 325 mesh (99\%), under the same experimental conditions. The width at half height, $\beta_{h k l}=\left(B_{h k l}-\right.$ b) was corrected, for each observed reflection with $\beta_{h k l}<1^{\circ}$, by subtracting the instrumental broadening of the closest silicon reflection from the experimental width at half height, $B_{h k l}$.

2.4.6. High resolution X-ray photoelectron spectroscopy (XPS). PHI 5000 VersaProbe instrument (Physical Electronics) was utilized for survey scan and high resolution X-ray photoelectron spectroscopy (XPS). The powder was dried in oven at $100{ }^{\circ} \mathrm{C}$ for $24 \mathrm{~h}$ at atmospheric pressure before analysis and thereafter placed in the XPS pre-chamber overnight, in order to avoid anomalous outgassing during the XPS characterization, performed in UHV condition (10-8 Pa). A monochromatic Al Kalpha X-ray source $(1486.6 \mathrm{eV}, 15 \mathrm{kV}$ voltage and $1 \mathrm{~mA}$ anode current) and a power of $25.2 \mathrm{~W}$ were used for analysis. Different pass energy values were employed: $187.85 \mathrm{eV}$ for survey spectra and $23.5 \mathrm{eV}$ for high resolution peaks. Analyses were carried out with a take-off angle of $45^{\circ}$ and with a $100 \mu \mathrm{m}$ diameter X-ray spot size on a square area of $1400 \times 1400 \mu \mathrm{m}^{2}$, with the aim to have a good average and better statistics of powder behavior. A double beam (electron and argon ion gun) neutralization system, dedicated to reduce the charging effect on samples, was also employed during data acquisition. All binding energies (B.E.) were referenced to the $\mathrm{C} 1 \mathrm{~s}$ line at $284.8 \mathrm{eV}$.

2.4.7. Cyclic voltammetry (CV). Electrochemical measurements cyclic voltammetry were conducted in a divided threeelectrode cell controlled by a Potentiostat Galvanostat model 2549 (Amel Instrument). A Pt reference and a Pt wire auxiliary electrode were used respectively throughout the work. A glassy carbon electrode (GC) was used as the working electrode. The electrodes were dried in air prior to electrochemical characterization. The potential was cycled from $-0.35 \mathrm{~V}$ to $+1.0 \mathrm{~V}$ at a scan rate of $0.1 \mathrm{~V} \mathrm{~s}^{-1}$ in an aqueous solution of $0.1 \mathrm{M} \mathrm{Et}_{4} \mathrm{NBF}_{4} /$ $10 \mathrm{mM} \mathrm{K}_{4} \mathrm{Fe}(\mathrm{CN})_{6}$. G-OH water suspension was $0.125 \mathrm{mM}$.

\subsection{Water suspensions of G-OH adducts}

2.5.1. Preparation. Water suspensions of G-OH at different concentrations $\left(1 \mathrm{mg} \mathrm{mL}{ }^{-1} ; 0.8 \mathrm{mg} \mathrm{mL}^{-1} ; 0.6 \mathrm{mg} \mathrm{mL}^{-1} ; 0.5 \mathrm{mg}\right.$ $\mathrm{mL}^{-1} ; 0.3 \mathrm{mg} \mathrm{mL}{ }^{-1} ; 0.2 \mathrm{mg} \mathrm{mL}^{-1} ; 0.1 \mathrm{mg} \mathrm{mL}^{-1} ; 0.05 \mathrm{mg} \mathrm{mL}^{-1}$; $0.025 \mathrm{mg} \mathrm{mL}^{-1} ; 0.01 \mathrm{mg} \mathrm{mL}^{-1}$ ) were prepared. Each suspension was sonicated for 15 minutes using an ultrasonic bath $(260 \mathrm{~W})$ and subsequently UV-Vis absorption was measured. The suspension $(10 \mathrm{~mL})$ of each sample was put in a Falcon ${ }^{\mathrm{TM}}(15$ $\mathrm{mL}$ ) and centrifuged at: $2000 \mathrm{rpm}$ for 5 minutes, $4000 \mathrm{rpm}$ for 
10 minutes, $4000 \mathrm{rpm}$ for 30 minutes and $4000 \mathrm{rpm}$ for 60 minutes. UV-Vis absorptions were measured immediately after each centrifugation and after 5 minutes, 10 minutes, 30 minutes, 60 minutes, 120 minutes, 4 days and 8 days.

2.5.2. UV-Vis spectroscopy. The suspensions of adduct ( $3 \mathrm{~mL}$ ) were placed by pipette Pasteur, in quartz cuvettes of $1 \mathrm{~cm}$ optical path (volume 1 or $3 \mathrm{~mL}$ ) and analyzed by a spectrophotometer Hawlett Packard 8452A Diode Array Spectrophotometer. It was recorded a white of the solvent employed. The UVvisible spectrum reported absorption as a function of the wavelength of the radiation between 200 and $750 \mathrm{~nm}$.

2.5.3. Dynamic light scattering (DLS). The nanoparticle size in G-OH suspensions was analyzed with a Zetasizer Dynamic Light Scattering system (Malvern Instrument Ltd.) at room temperature using an $1 \frac{1}{4} 632.8 \mathrm{~nm} \mathrm{He}-\mathrm{Ne}$ laser. Each centrifuged suspension, prepared using a concentration of $1 \mathrm{mg}$ $\mathrm{mL}^{-1}$, was analyzed by DLS.

2.5.4. High-resolution transmission electron microscopy (HRTEM). HRTEM investigations on G-OH samples taken from the sonicated suspensions were carried out with a Philips CM 200 field emission gun microscope operating at an accelerating voltage of $200 \mathrm{kV}$. Few drops of the water suspensions were deposited on 200 mesh lacey carbon-coated copper grid and airdried for several hours before analysis. During acquisition of HRTEM images, the samples did not undergo structural transformation. Low beam current densities and short acquisition times were adopted. To estimate the number of stacked graphene layers and the dimensions of the stacks visible in HRTEM micrographs, the Gatan Digital Micrograph software was used.

2.5.5. Preparation of few layer graphene by centrifugation of G-OH water dispersions. Water suspension of G-OH at $1 \mathrm{mg}$ $\mathrm{mL}^{-1}$ was first prepared $100 \mathrm{mg}$ in $100 \mathrm{~mL}$ of water and after sonicated for 30 minutes using an ultrasonic bath $(260 \mathrm{~W})$. The so obtained suspension was put in a Falcon ${ }^{\mathrm{TM}}(10 \mathrm{~mL} \times 10$ times) and centrifuged at $2000 \mathrm{rpm}$ for 10 minutes. After centrifugation supernatant was removed and the water was removed at reduced pressure.

The yield of few-layer graphene (FLG) was determined by analysing the weight percentage of all of exfoliated flakes after separation by centrifugation.

\subsection{Thin films of HSAG and G-OH on paper substrate}

2.6.1. Preparation of G-OH thin films. A water suspension of $\mathrm{G}-\mathrm{OH}\left(1 \mathrm{mg} \mathrm{mL}{ }^{-1}\right)$ was first prepared and about $1 \mathrm{~mL}$ was deposited on the top of the paper by a syringe and spread by a bar coater (K Control Coater 101) with a close wound bar (wet film deposited $6 \mathrm{~mm}$ ) at $7.5 \mathrm{~m} \mathrm{~min}^{-1}$.

2.6.2. Preparation of HSAG thin films. HSAG (5 mg) was first premixed with carboxymethylcellulose sodium $(10 \%$ by mass) and a water suspension $\left(1 \mathrm{mg} \mathrm{mL}^{-1}\right)$ was prepared. About $1 \mathrm{~mL}$ was deposited on the top of the paper by a syringe and spread by a bar coater (K Control Coater 101) with a close wound bar (wet film deposited $6 \mathrm{~mm}$ ) at $7.5 \mathrm{~m} \mathrm{~min}^{-1}$.

2.6.3. Four-point probe method. Direct current (DC) electrical conductivity $(\sigma)$ was measured by the four point probe (FPP) method $^{31}$ by using a hand applied FPP device (Jandel
Engineering Ltd., UK) with a probe head with linear arrayed tungsten carbide needles (tip radii $300 \mathrm{~mm}$, needles spacing $635 \mathrm{~mm}$, loads $60 \mathrm{~g}$ ) coupled with a Keithley 2601 electrometer. Data were acquired and analyzed by CSM/Win Semiconductor Analysis Program software (MDC, US).

\section{Results and discussion}

Two methods were adopted for the introduction of oxygenated functional groups on HSAG: the well known Staudenmaier method, based on graphite oxidation with $\mathrm{KClO}_{4}$, in the presence of a mixture of nitric and sulphuric acid, ${ }^{13}$ and the reaction of graphite with $\mathrm{KOH} .{ }^{32}$ The former preparation and the characterization of GO obtained therefrom have been reported elsewhere by one of the authors. ${ }^{30}$ The preparation of hydroxyl functionalized HSAG, from the reaction with $\mathrm{KOH}$, is described as follows. Such reaction was performed with the help of either mechanical or thermal energy, obtaining G-OH-M and G-OH-T, respectively. Thermal and mechanical energy were also used in sequence, preparing G-OH-TM. Procedures are described in the experimental part and are summarized in the block diagram in Fig. 1.

Preparation of G-OH via mechanical treatment was carried out adopting two $\mathrm{KOH} / \mathrm{HSAG}$ ratios: about $20: 1$ and $10: 1$ (method $\mathrm{A}$ and method $\mathrm{B}$, respectively, in the experimental part). In this paper, samples obtained with method B are discussed. G-OH samples were washed until neutral pH of washing water. Characterization of three representative samples, from thermal, mechanical and thermal-mechanical treatments, is discussed as follows.

Chemical composition of the samples was investigated by means of elemental analysis, TGA, XPS and FTIR analysis.

Results from elemental and thermogravimetric analysis and thermographs of HSAG and G-OH-M are reported as ESI. $\dagger$ Data from elemental analysis are in Table S.1. $\dagger$ Pristine HSAG was found to have the following chemical composition (\% by mass): carbon 96.8, oxygen 4.2. The oxygen content of G-OH adducts, as expected, increased to about 5.0 and 6.1, for G-OH-T and G-OH-M, respectively. Largest amount of oxygen from 9 to $16 \%$ was introduced by adopting larger $\mathrm{KOH} / \mathrm{HSAG}$ ratio (method A) for the mechanical treatment. Method B, based on lower $\mathrm{KOH}$ amount, was used in order to modify pristine HSAG only to a minor extent.

TGA revealed lower mass loss for HSAG, below $700{ }^{\circ} \mathrm{C}$, than G-OH samples, as it is shown by data in Table $\mathrm{S} .2$ and by comparison of traces in Fig. S.1 (HSAG) and S.2-S.4† (G-OH). For G-OH samples, a three-step decomposition profile can be observed. The first mass loss below $150{ }^{\circ} \mathrm{C}$ can be attributed to the water removal. The second and third mass losses, that are due to decomposition of oxygen-containing groups and removal of alkenylic groups, occur in the temperature range between $150{ }^{\circ} \mathrm{C}$ and $700{ }^{\circ} \mathrm{C}$. Combustion with oxygen of graphite occurs for temperatures higher than $700^{\circ} \mathrm{C}$. The low mass loss of HSAG below $700{ }^{\circ} \mathrm{C}$ indicates the presence of a minor amount of sorbed water and of functional groups that can undergo degradation. For G-OH samples, the larger amount of mass loss at $T>150{ }^{\circ} \mathrm{C}$ is accompanied by larger mass loss at $T<150^{\circ} \mathrm{C}$. It 


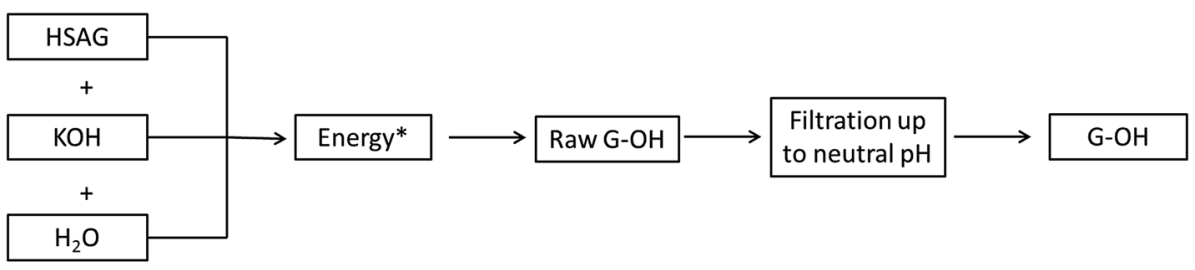

*mechanical or thermal

Fig. 1 Block diagram for the preparation of $\mathrm{G}-\mathrm{OH}$ adducts: by either thermal (G-OH-T) or mechanical energy (G-OH-M).

could be hypothesized that G-OH containing larger amount of oxygenated functional groups holds larger amount of water. It is finally worth observing that the onset temperature of the last degradation step becomes appreciably lower in G-OH samples than in HSAG sample, presumably as a consequence of combustion of edge carbons with oxygen-containing functional groups.

Type and amount of atoms and functional groups in pristine HSAG and in G-OH were then studied through wide scan XPS spectra (Fig. 2a). As expected, in both spectra the main signals are due to $\mathrm{C} 1 \mathrm{~s}$ and $\mathrm{O} 1 \mathrm{~s}$ (binding energy B.E. $285 \mathrm{eV}$ and $532 \mathrm{eV}$ respectively). After treatment with $\mathrm{KOH}$ the modified graphite shows also two small noisy signal at B.E. around $400 \mathrm{eV}$ and 385 $\mathrm{eV}$, attributable to $\mathrm{N} 1 \mathrm{~s}$ and $\mathrm{K} 2 \mathrm{~s}$. The $\mathrm{O} 1 \mathrm{~s} / \mathrm{C} 1 \mathrm{~s}$ atomic ratio of the pristine graphite is 0.04 (slightly higher than the 0.03 value reported in the literature for graphite), corresponding to an atomic $\mathrm{O}$ percentage of $4.2 \%$. Treatment of $\mathrm{KOH}$ affects the oxygen content: in G-OH its atomic percentage is in fact $6.4 \%$, and the $\mathrm{O} 1 \mathrm{~s} / \mathrm{C} 1 \mathrm{~s}$ ratio increases to 0.07 .

Narrow scan spectra for each element were recorded, C 1s and $\mathrm{O} 1 \mathrm{~s}$ envelopes were evidenced and different contributions were identified by a curve fitting processing. As shown in Fig. 2b, C 1s (main peak at B.E. $=284.8 \mathrm{eV}$ ) of both samples have a broad, asymmetric tail towards higher binding energy and one satellite signal, few eV from the main peak, related to the $\pi-\pi^{*}$ transition (shake-up at around $290.9 \mathrm{eV}$ ): these two features are found when a high concentration of $\mathrm{sp}^{2}$ carbon is present. In the envelopes of $\mathrm{C} 1 \mathrm{~s}$, the peak centered at $284.8 \mathrm{eV}$ is related to $\mathrm{C} \mathrm{sp}^{2}$; the other peaks at $285.3 \mathrm{eV}$ and $287.3 \mathrm{eV}$ are likely to characterize $\mathrm{C}-\mathrm{O}$ and $\mathrm{C}=\mathrm{O}$ functions and their intensity is changed by the $\mathrm{KOH}$ treatment, although one can not exclude the presence of $\mathrm{C}-\mathrm{H}$ environments and other defects. The relative portion of these functions, i.e. the $\mathrm{C}-\mathrm{O} / \mathrm{C}=\mathrm{O}$ ratio, changes from 2.2 to 5.4, which means that the amount of $\mathrm{C}-\mathrm{O}$ groups increases with the functionalization reaction. These data are confirmed by the analysis of the $\mathrm{O} 1 \mathrm{~s}$ envelope: on both samples, in fact, two main peaks are present, corresponding to C-O type oxygen at 533.1 eV (hydroxylic groups, non-carbonyl ether type - oxygen atoms in esters and anhydrides) and to $\mathrm{C}=\mathrm{O}$ type oxygen at $531.1 \mathrm{eV}$ (carbonylic oxygen in esters and anhydrides)..$^{33}$

Functional groups present in HSAG, G-OH and GO samples were investigated with IR measurements on samples prepared in a Diamond Anvil Cell (DAC). In Fig. 3 the IR spectra of HSAG (a), G-OH-T (b), G-OH-TM (c), G-OH-M (d) and GO from Staudenmaier oxidation (e) are reported.

All the spectra in Fig. 3 are characterized by an increasing background toward high wavenumbers due to diffusion/reflection phenomena of the IR light by the particles of the sample. The spectrum of HSAG (Fig. 3a) is characterized by the band at
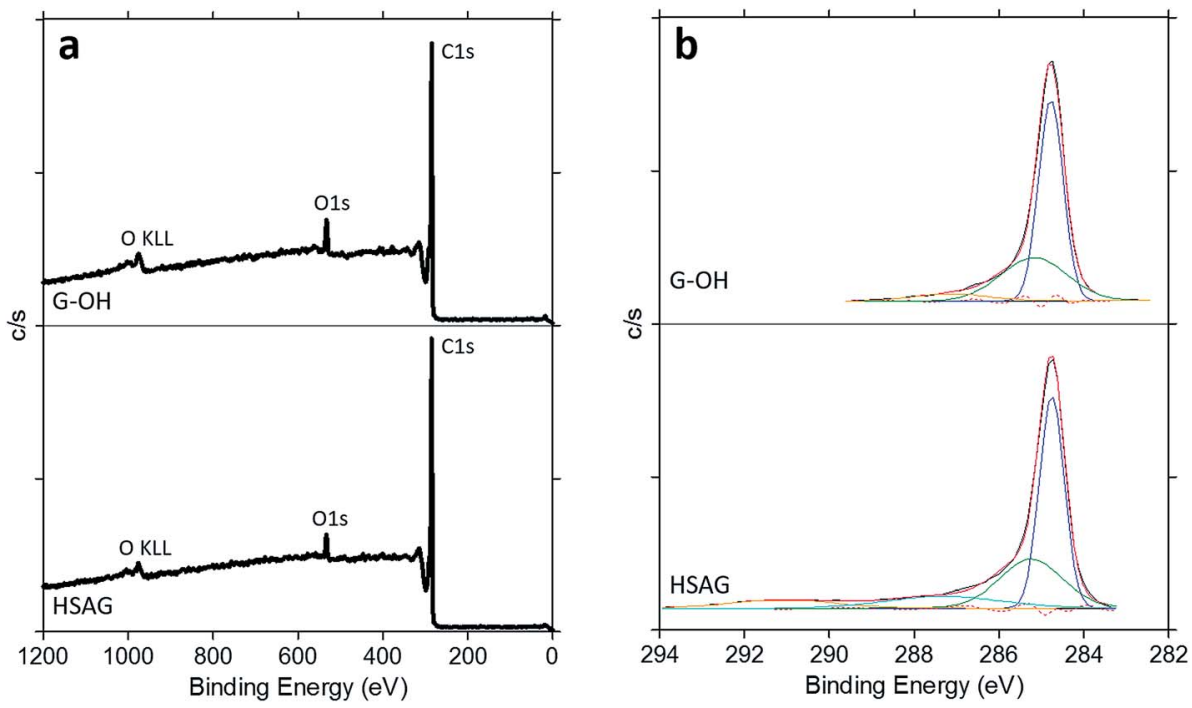

Fig. 2 Wide scan XPS spectra (a) and high resolution analysis of $\mathrm{C}$ 1s region (b) of $\mathrm{HSAG}$ and G-OH. 


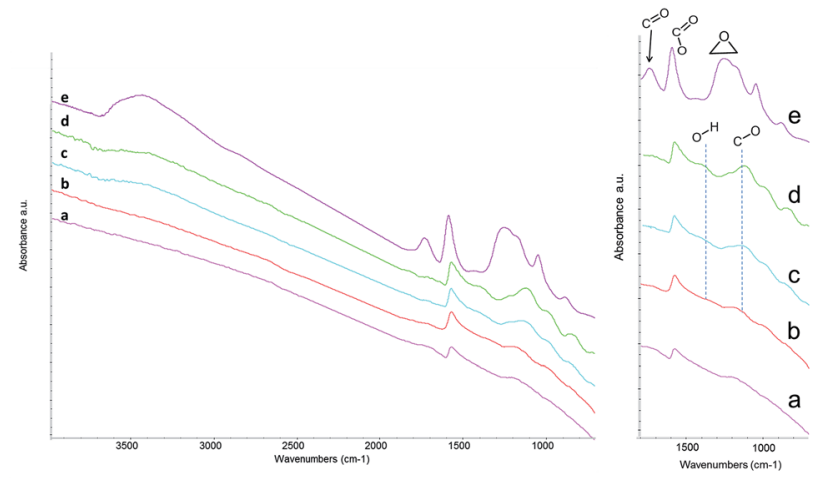

Fig. 3 IR spectra of HSAG (a), G-OH-T (b), G-OH-TM (c), G-OH-M (d) and $\mathrm{GO}(\mathrm{e})$.

$1590 \mathrm{~cm}^{-1}$ which is the peak of graphite and graphene materials, assigned to $E_{1 u}$ IR active mode of collective $C=C$ stretching vibration. In the spectra of G-OH samples (Fig. 3b-d) the $\mathrm{C}=\mathrm{C}$ peak is still observed along with new bands. These new bands can be assigned to the absorption of different $-\mathrm{OH}$ groups bonded to the graphene sheets. The corresponding peaks are located at: (i) $3400 \mathrm{~cm}^{-1}$, assigned to the $-\mathrm{OH}$ stretching vibrations of hydrogen bonded hydroxyl groups; (ii) $1388 \mathrm{~cm}^{-1}$, assigned to the out of plane vibration -OH groups; (iii) $1110 \mathrm{~cm}^{-1}$, assigned to $\mathrm{C}-\mathrm{O}$ stretching vibration and (iv) $970 \mathrm{~cm}^{-1}$, assigned to the in plane phenyl-O-H bending.

The spectrum of GO (Fig. 3e) shows peculiar features different from G-OH samples. The band at $3400 \mathrm{~cm}^{-1}(-\mathrm{OH}$ stretching) is broad and stronger. Carboxylic groups originates the large and strong band located at $1727 \mathrm{~cm}^{-1}$. The $\mathrm{C}=\mathrm{C}$ peak becomes very strong due to the polarizing effect of the $-\mathrm{C}=\mathrm{O}$ groups bonded to the graphene backbone. Other strong and broad features occurring at $1250 \mathrm{~cm}^{-1}$ and $877 \mathrm{~cm}^{-1}$ can be assigned to the ring breathing and the $\mathrm{C}-\mathrm{O}-\mathrm{C}$ bond stretching of epoxide groups respectively, together with other vibrations of $\mathrm{C}-\mathrm{O}-\mathrm{C}$ and $-\mathrm{OH}$ functionalities. The peak at $1044 \mathrm{~cm}^{-1}$ could be ascribed to the stretching vibration of $\mathrm{S}=\mathrm{O}$ bonds. It is worth noticing that the IR spectrum of GO from Staudenmaier method (Fig. 3e) shows the presence of oxygenated functional groups, mainly carboxyl and epoxide groups, that are not present on G$\mathrm{OH}$ samples.

Structure of HSAG and G-OH were studied by means of WAXD, Raman and TEM analyses.

Fig. 4 shows WAXD patterns taken on powders of HSAG (Fig. 4a), G-OH-T (Fig. 4b), G-OH-M (Fig. 4c) and G-OH-TM (Fig. 4d). Moreover, it is shown the pattern of a GO sample obtained through the Staudenmaier method (Fig. 4e).

In pristine HSAG, crystalline order in the direction orthogonal to structural layers is revealed by two (00l) reflections: 002 at $26.6^{\circ}$, that corresponds to an interlayer distance of $0.338 \mathrm{~nm}$ and 004 at $54.3^{\circ}$. Such interlayer distance is slightly larger than the one of ordered graphite samples $\left(d_{002}=0.335 \mathrm{~nm}\right) .{ }^{34}$ The in plane order is shown by 100 and 110 reflections, at $42.5^{\circ}$ and $77.6^{\circ}$ respectively. By applying the Scherrer equation (see the Experimental section) to (002) and (110) reflections, respectively, the out of plane $\left(D_{\perp}\right)$ and the in plane $\left(D_{\|}\right)$correlation

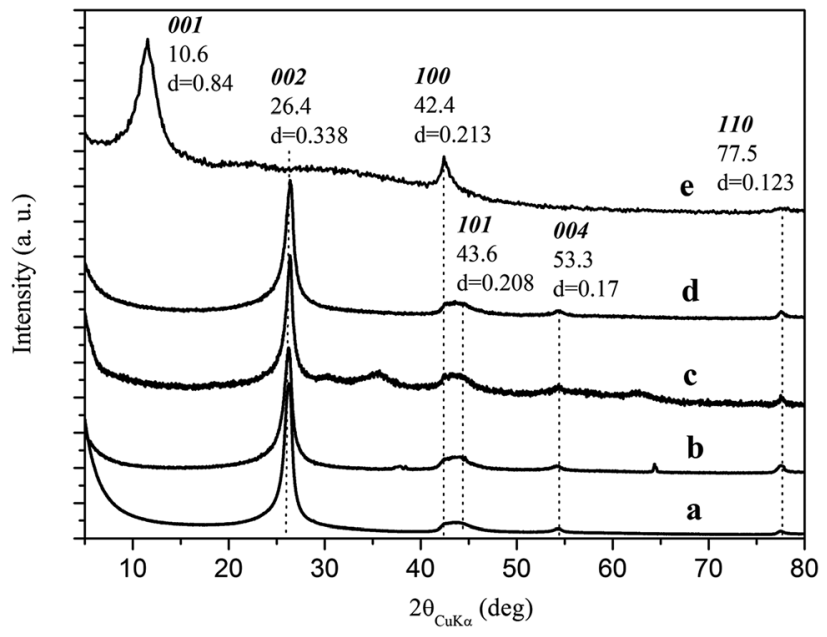

Fig. 4 WAXD patterns of HSAG (a), G-OH-T (b), G-OH-M (c), G-OHTM (d) and GO (e).

lengths were calculated. Values were $9.8 \mathrm{~nm}$ for $\left(D_{\perp}\right)$ and 30.2 $\mathrm{nm}$ for $\left(D_{\|}\right)$. The in-plane correlation length is thus larger than the out-of-plane correlation length. From the values of $\left(D_{\perp}\right)$ and of the interlayer distance, the number of stacked layers was estimated to be about 35 . These results reveal the turbostratic nature of HSAG ${ }^{30}$ that has however remarkable crystalline order inside the structural layers. HSAG appears thus a good graphite grade for the preparation of conductive coating layers, provided that the $\mathrm{sp}^{2}$ nature of carbon atoms is not perturbed.

(002) reflection in the patterns of G-OH samples remains at the same $2 \theta$ value, indicating that the oxidation reaction did not promote expansion of the interlayer distance. There is, instead, a large change in the position of $(00 l)$ reflections in the pattern of GO from oxidation with the Staudenmaier method. (001) reflection is at $2 \theta=10.45$ and corresponds to an average interlayer spacing of $0.9 \mathrm{~nm}$. These differences are justified by the presence of oxygen-containing functional groups attached on both sides of the graphene sheet. The number of stacked layers in G-OH samples was calculated by applying the Scherrer equation to 002 reflection and was found to be between 20 and 25. 100 and 110 reflections, due to the in plane order, are clearly visible in G-OH patterns. From these findings, it can be commented that the reaction with $\mathrm{KOH}$ promotes the partial exfoliation of HSAG without altering the in plane order.

Raman spectroscopy is widely employed for the study of carbonaceous materials. ${ }^{35-41}$ In Raman spectra, two peaks, named $\mathrm{D}$ and $\mathrm{G}$ and located at $1350 \mathrm{~cm}^{-1}$ and $1590 \mathrm{~cm}^{-1}$ respectively, are in particular investigated. The G peak is due to bulk crystalline graphite (graphene), whereas the $\mathrm{D}$ peak appears in the presence of either structural defects, such as holes, $\mathrm{sp}^{3}$ or $\mathrm{sp}$ carbon atoms, free radicals, distortions from planarity, grafted functional groups or confinement (e.g. by edges) of the graphitic layers. ${ }^{38-41}$ It is worth reminding that graphitic layers have finite dimensions and irregular boundaries with, for example, dangling bonds. Graphitic powders that experienced ball milling, as HSAG, revealed in the Raman spectrum evident D line, whose intensity increased with the 
grinding time. ${ }^{41}$ In this work, Raman spectroscopy was applied to the structural characterization of HSAG and G-OH-M, and the spectra are shown in Fig. 5a and in Fig. 5b, respectively. Analysis was performed on a good number of spots and spectra can be considered representative of the investigated samples.

$\mathrm{G}$ and $\mathrm{D}$ bands are present in both spectra, with similar intensity. The high intensity of the $\mathrm{D}$ band can be interpreted taking into account what reported in the literature for Raman spectra of ball milled graphite. ${ }^{\mathbf{4 2}}$ The electronic and vibrational properties of a core of graphitic samples sufficiently far from the edge can not be distinguished from those of infinite and ideal graphene layers. A crown confined region, close to the edge, is electronically perturbed and the electronic and vibrational structures are different from those of the bulk materials. The pronounced $\mathrm{D}$ band is thus due to the different types of molecular disorder. However, there are not indications that the reaction of HSAG with $\mathrm{KOH}$ appreciably alter the structure of the graphitic layers.

The structure of G-OH adduct was investigated also by performing HRTEM analysis on samples isolated from water supernatant suspensions (see below in the text for the discussion on water suspension), after centrifugation for $10 \mathrm{~min}$ at $2000 \mathrm{rpm}$ and for $15 \mathrm{~min}$ at $9000 \mathrm{rpm}$. Fig. 6 shows HR-TEM micrographs at lower and higher magnifications.

Micrographs at lower magnification in Fig. 6a and c reveal that the lateral size of G-OH adducts is of the same order of magnitude in samples isolated after centrifugations at different rpm and for different times. Hence, there is not indication that the milling step cause appreciable breaking of the graphitic layers. Micrographs at higher magnification allow to visualize the stacks of graphene layers that are disposed with a lateral side perpendicular to the beam. Thanks to this disposition of the nano-stacks, it is possible to estimate the number of stacked graphene layers. Fig. $6 \mathrm{~b}$ shows stacks isolated after centrifugation for $10 \mathrm{~min}$ at $2000 \mathrm{rpm}$ : are visible stacks (indicated in the boxes) of about 1.7-4.8 nm, with 6 to 15 stacked graphene

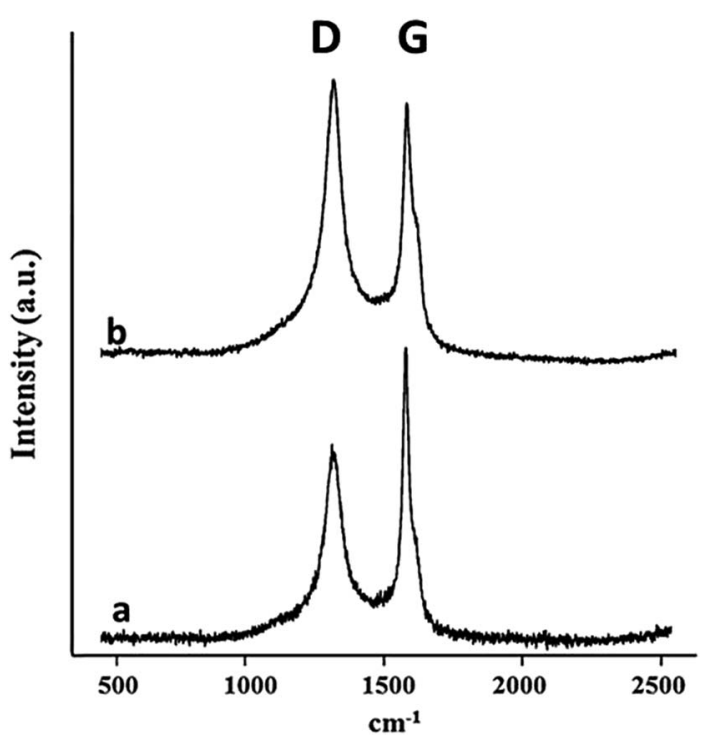

Fig. 5 Raman spectra of HSAG (a) and G-OH-M (b).

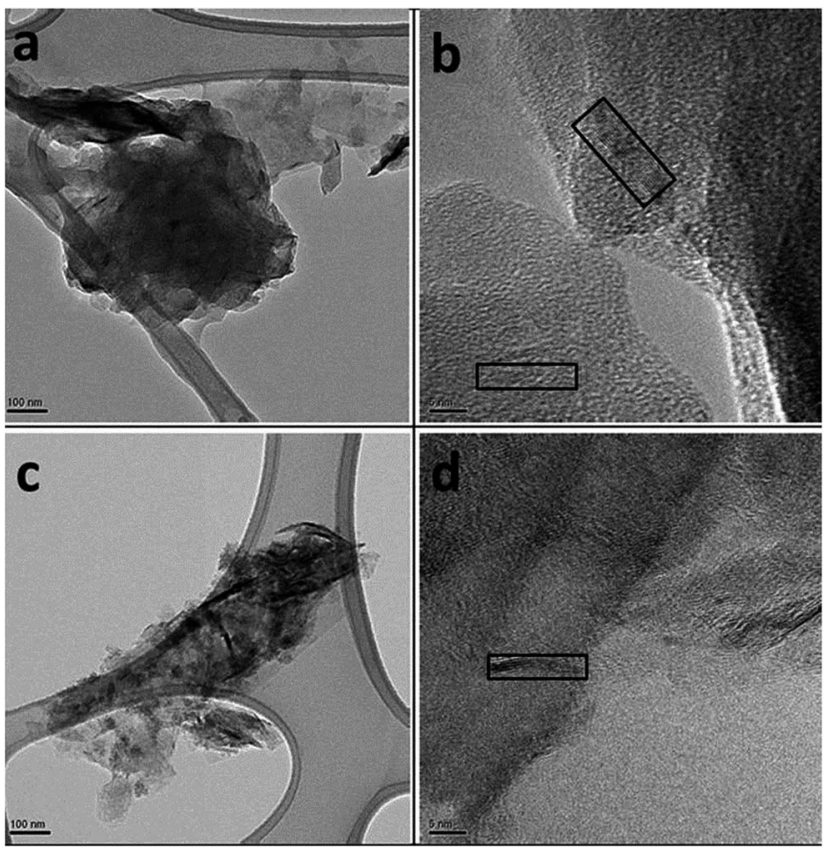

Fig. 6 Micrographs of $\mathrm{G}-\mathrm{OH}-\mathrm{M}$ adduct isolated from supernatant suspension, after centrifugation for $10 \mathrm{~min}$ at $2000 \mathrm{rpm}$ (a, b), $15 \mathrm{~min}$ at 9000 rpm (c, d). Micrographs are: low magnification bright field TEM $(a, c)$, HRTEM images (b, d).

layers. As revealed by the exam of a good number of micrographs, such stacks are the most abundant ones in a stacks' population that contains either little larger of little lower number of layers. Fig. 6d shows a stack (indicated in the box) isolated after centrifugation for $15 \mathrm{~min}$ at $9000 \mathrm{rpm}$ : is of about $1.7 \mathrm{~nm}$ and contains about 6 stacked graphene layers. Such a stack was very frequently observed in the analyzed micrographs after centrifugation for 15 minutes.

Reaction of high surface area nanosized graphite with $\mathrm{KOH}$ allows thus to introduce an appreciable amount of hydroxyl functional groups (from 4 to $6 \%$ by mass), promoting partial exfoliation but maintaining the same interlayer distance between the graphene layers, without altering the bulk crystalline order of the graphitic layers. It appears thus a reasonable hypothesis that hydroxyl groups are located on the edge carbon atoms. A mechanistic pathway can be hypothesized for this reaction. The insertion of hydroxyl anion can occur on peripheral carbon and the following two step mechanism can be hypothesized: (i) a nucleophilic initial addition of an hydroxide ion to the peripheral aromatic ring followed by (ii) re-aromatization processes. In the frame of this mechanism, a negatively charged intermediate would be formed. Such intermediate would exist in a considerable number of resonance hybrids. Such large stabilization could be considered a sort of "wave effect". Driving force would be the aromatization of the graphene layer (Fig. S.5†).

Dispersions of G-OH in water were prepared through a mild sonication. Results obtained with G-OH-TM are reported as follows. Dispersions were with different concentrations $(0.01$, 
0.02, 0.05, 0.1, 0.2, 0.3, 0.5, 1.0 and $4.0 \mathrm{mg} \mathrm{mL}^{-1}$ ). Fig. 7 shows dispersions in the range from 0.01 to $1 \mathrm{mg} \mathrm{mL}^{-1}$.

Fig. 8 reports results from UV-Vis absorption analysis. Fig. 8A shows that the absorbance monotonously increases with G-OHTM concentration and Fig. S.6† demonstrates a linear correlation between absorption and G-OH-TM concentration, thus revealing that water suspension of G-OH-TM were prepared. Fig. 8B shows that the absorbance detected for the freshly prepared suspension ( $\left.1 \mathrm{mg} \mathrm{mL}{ }^{-1}\right)$ did not change after 1 week storage. Traces in Fig. $8 \mathrm{C}$ indicate that the centrifugation at $2000 \mathrm{rpm}$ for 10 minutes led to a slight decrease of absorbance.

Stability of G-OH-TM suspension was verified also at a concentration of $4 \mathrm{mg} \mathrm{mL} \mathrm{m}^{-1}$, after storage for 4 weeks.

Average size of G-OH-M and G-OH-T aggregates in water suspensions was investigated by means of dynamic light scattering (as reported in the experimental part). Table 1 shows the average size of particles, for water suspensions of HSAG and of both the adducts.

Introduction of oxygenated functional groups leads to the reduction of HSAG aggregates size. G-OH particles in the supernatant suspensions (both from mechanical and thermal treatments) appear to have lower average size than particles of HSAG and GO. In particular, the mechanical treatment appears to promote the formation of smaller G-OH aggregates. It could be also commented that the presence of large amount of functional groups can favour the aggregation of GO particles.

Preparation of G-OH water suspension was investigated as a method for producing few layer graphene. As described in the experimental part, $1 \mathrm{mg} \mathrm{mL}^{-1} \mathrm{G}-\mathrm{OH}$ suspension was centrifuged at $2000 \mathrm{rpm}$ for 10 minutes, obtaining a supernatant suspension containing stacks of graphene with layer number ranging from 6 to 15, as observed via HRTEM and confirmed by WAXD analysis. The amount of few layer graphene in the supernatant suspension was about $35-40 \%$.

As mentioned in the introduction, objective of this work was the preparation of flexible and electrically conductive carbon papers by deposing G-OH suspensions on different substrates, avoiding the reduction step. G-OH layer was deposed on paper using a bar coater (as described in the experimental part, with 6 , 12, 24, $40100 \mu \mathrm{m}$ bars), obtaining flexible carbon paper. Two types of suspensions were mainly investigated: $1 \mathrm{mg} \mathrm{mL}^{-1}$ suspension as prepared by sonication and suspension after

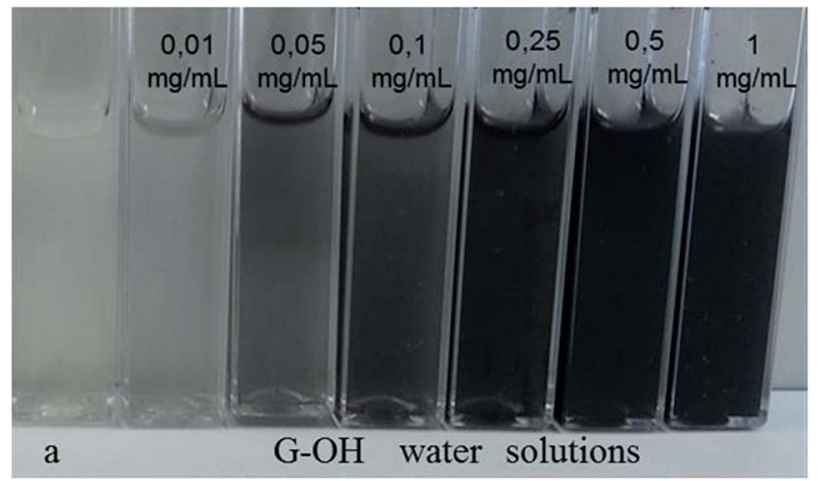

Fig. 7 G-OH-TM water dispersions at different concentration.
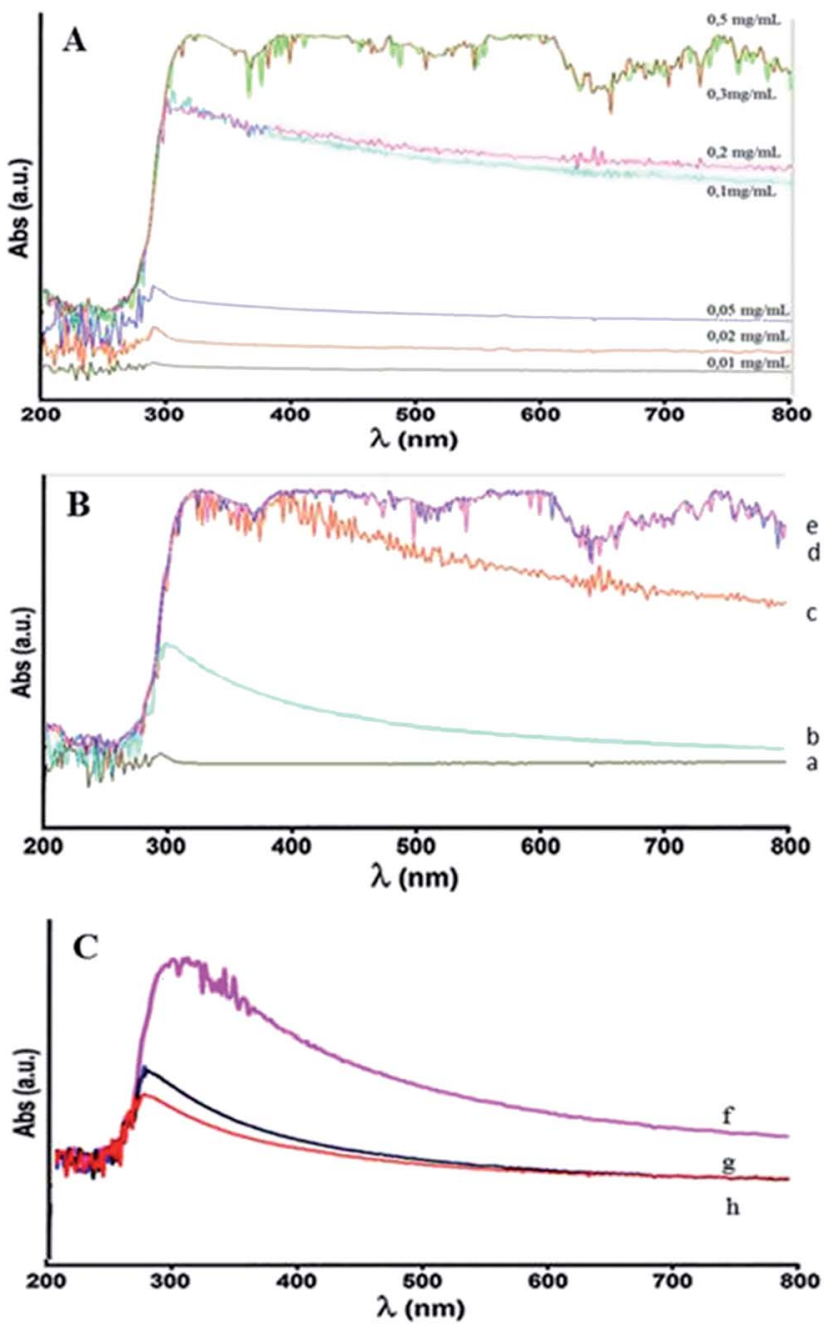

Fig. 8 Dependence of UV-Vis absorbance on concentration of G-OH water suspensions (A) and UV-Vis spectra of graphite in water (a), UVVis spectra of G-OH-TM after centrifugation at 4000 rpm for 1 hour (b), after centrifugation at $2000 \mathrm{rpm}$ for $10 \mathrm{~min}$ (c), after sonication (d) and after 1 week (e) (B), and UV-Vis spectra of GO Staudenmaier after centrifugation at $9000 \mathrm{rpm}$ for 5 minutes (f), for 30 minutes (g), for 60 minutes (h) (C).

Table 1 Average size of HSAG, G-OH-M, G-OH-T and GO determined through DLS

\begin{tabular}{lll}
\hline & \multicolumn{2}{c}{ Average size of particles $(\mathrm{nm})$} \\
\cline { 2 - 3 } & Starting suspension & Supernatant suspension $^{a}$ \\
\hline HSAG & 500 & 194 \\
G-OH-M & 340 & 140 \\
G-OH-T & 470 & 190 \\
GO & 380 & 250 \\
${ }^{a}$ After centrifugation at 9000 rpm for 1 hour.
\end{tabular}

centrifugation at $2000 \mathrm{rpm}$ for 10 minutes. Structure of the graphitic coating on paper was studied by means of WAXD analysis. Coating layers obtained with both types of 
suspensions revealed a number of graphene layers (evaluated from peak shape analysis, as reported above) close or higher than that detected on pristine G-OH. In Fig. S.7† are reported patterns of G-OH-M (a), paper support (b) and graphitic coating layer (c). $D_{002}$ correlation length of the graphitic layer of about $17 \mathrm{~nm}$ (corresponding to about 50 stacked layers) was calculated. This result could be expected, as the coating procedure favors the stacking of graphene layers and indicates that the key aspect for the preparation of few layers graphene coating is the selection of the starting suspension: supernatant suspension after centrifugation should be used.

Electrical conductivity of G-OH coated paper was measured with the four point probe, as described in the experimental part, and data are shown in Table 2.

Very high resistivity was measured for the GO layer from Staudenmaier oxidation, as expected. In fact, only the reduction step is able to restore, though if not completely, the $\mathrm{sp}^{2}$ hybridization of the carbon atoms. Conductive HSAG layer was not obtained, probably as a consequence of the large amount of binder (carboxymethylcellulose) needed to disperse HSAG. The electrical conductivity detected in the G-OH layer confirms that carbon atoms have largely prevailing $\mathrm{sp}^{2}$ hybridization, as HSAG functionalization occurred on peripheral positions. Defects on the edges of graphene layers in pristine HSAG and hydroxyl functionalization can be considered to adversely affect the electrical conductivity of carbon papers.

Electrical properties of G-OH were also investigated by cyclic voltammetry. In the literature, $\mathrm{CV}$ was used to show the good electroactivity of $\mathrm{G}-\mathrm{OH}$ (from ball milling with $\mathrm{KOH}$ ). ${ }^{32}$ Peak separation, measured for the $\left[\mathrm{Fe}(\mathrm{CN})_{6}\right]_{4} /\left[\mathrm{Fe}(\mathrm{CN})_{6}\right]_{3}$ redox pair, was found to significantly decrease when the glassy carbon electrode was coated with G-OH and this was taken as indication of significant increase of electron transfer rate. This CV experiment and the above reported four point measurements show that $\mathrm{G}-\mathrm{OH}$ with substantially unaltered graphitic structure is electrically active. In this work, $\mathrm{CV}$ measurements were taken (graphs are in Fig. S.8†) on $0.1 \mathrm{M} \mathrm{Et}_{4} \mathrm{NBF}_{4} / 10 \mathrm{mM} \mathrm{K}_{4} \mathrm{Fe}(\mathrm{CN})_{6}$ solution (a), obtaining the expected cyclic voltammogram, and on a $0.125 \mathrm{mM}$ G-OH suspension/0.1 $\mathrm{M} \mathrm{Et}_{4} \mathrm{NBF}_{4}$ (b), without observing any detectable signal, as it should be expected for a graphite with very minor amount of oxygenated functional groups. On the contrary, cyclic voltammograms are reported in the literature for graphite oxides obtained through standard oxidation methods ${ }^{43}$ that means GO with remarkable amount of functional groups, such as GO from Staudenmeier oxidation. These findings are in line with the other results of this work: $\mathrm{OH}$ functionalization allows the preparation of water graphite suspensions and of carbon papers, without affecting graphitic

Table 2 Electrical conductivity of HSAG, G-OH-M and graphite oxide (GO)

\begin{tabular}{|c|c|c|c|}
\hline Nano-graphite sample & HSAG $^{a}$ & G-OH-M & $\mathrm{GO}^{b}$ \\
\hline Electrical conductivity ${ }^{c}\left(\mathrm{~S} \mathrm{~m}^{-1}\right)$ & $9 \times 10^{-4}$ & $4.4 \times 10^{-3}$ & $7 \times 10^{-}$ \\
\hline
\end{tabular}

${ }^{a}$ Carboxymethyl-cellulose was used to obtain the film. ${ }^{b}$ By
Staudenmaier method (ref. 30). ${ }^{c} 100 \mu \mathrm{m}$ thick.

\section{Acknowledgements}

Authors gratefully acknowledge Dr Mario Maggio (Università degli Studi di Salerno) for X-ray analysis, Prof Elena Vismara (Politecnico di Milano) for cyclic voltammetry and Dr Salvatore Guastella (Politecnico di Torino) for XPS measurements, and 
are in debt to Prof. Attilio Citterio for many useful discussions. Financial support was from "PRIN Research Project 20102011".

\section{References}

1 K. S. Novoselov, A. K. Geim, S. V. Morozov, D. Jiang, Y. Zhang, S. V. Dubonos, I. V. Grigorieva and A. A. Firsov, Electric field effect in atomically thin carbon films, Science, 2004, 306, 666-669.

2 A. K. Geim and A. H. MacDonald, The rise of graphene, Phys. Today, 2007, 60, 35.

3 A. Peigney, C. H. Laurent, E. Flahaut, R. Bacsa and A. Rousset, Specific surface area of carbon nanotubes and bundles of carbon nanotubes, Carbon, 2001, 39, 507-514.

4 Y. Zhang, J. W. Tan, H. L. Stormer and P. Kim, Experimental observation of the quantum Hall effect and Berry's phase in graphene, Nature, 2005, 438, 201-204.

5 E. T. Thostenson, C. Y. Li and T. W. Chou, Nanocomposites in context, Compos. Sci. Technol., 2005, 65, 491.

6 S. Stankovich, D. A. Dikin, G. H. B. Dommett, K. M. Kohlhaas, E. J. Zimney, E. A. Stach, R. D. Piner, S. T. Nguyen and R. S. Ruoff, Graphene-based composite materials, Nature, 2006, 442, 282.

7 C. Lee, X. Wei, J. W. Kysar and J. Hone, Measurement of the elastic properties and intrinsic strength of monolayer graphene, Science, 2008, 321, 385-388.

8 A. K. Geim, Graphene: status and prospects, Science, 2009, 324(5934), 1530-1534.

9 L. Qu, Y. Liu, J. B. Baek and L. Dai, Nitrogen-doped graphene as efficient metal-free electrocatalyst for oxygen reduction in fuel cells, ACS Nano, 2010, 4, 1321.

10 P. Sutter, Epitaxial graphene: How silicon leaves the scene, Nat. Mater., 2009, 8, 171.

11 S. Bae, H. Kim, Y. Lee, X. Xu, J. Park, Y. Zheng, J. Balakrishnan, T. Lei, H. R. Kim, Y. I. Song, Y. J. Kim, K. S. Kim, B. € Ozyilmaz, J. H. Ahn, B. H. Hong and S. Iijima, Roll-to-roll production of 30-inch graphene films for transparent electrodes, Nat. Nanotechnol., 2010, 5, 574.

12 X. Li, W. Cai, J. An, S. Kim, J. Nah, D. Yang, R. Piner, A. Velamakanni, I. Jung, E. Tutuc, S. K. Banerjee, L. Columbo and R. S. Ruoff, Large-area synthesis of highquality and uniform graphene films on copper foils, Science, 2009, 324, 1312.

13 L. Staudenmaier, Verfahren zur darstellung der graphitsäure, Ber. Dtsch. Chem. Ges., 1898, 31, 1481-1487.

14 W. S. Hummers and R. E. Offeman, Preparation of graphitic oxide, J. Am. Chem. Soc., 1958, 80, 1339.

15 C. Hontoria-Lucas, A. J. Lopez-Peinado, J. D. LopezGonzaiez, M. L. Rojas-Cerantes and R. M. Martin-Aranda, Study of oxygen-containing groups in a series of graphite oxides: physical and chemical characterization, Carbon, 1995, 33, 1585-1592.

16 D. C. Marcano, D. V. Kosynkin, J. M. Berlin, A. Sinitskii, Z. Sun, A. Slesarev, L. B. Alemany, W. Lu and J. M. Tour, Improved synthesis of graphene oxide, ACS Nano, 2010, 4(8), 4806-4814.
17 L. Sun and B. Fugetsu, Mass production of graphene oxide from expanded graphite, Mater. Lett., 2013, 109, 207-210.

$18 \mathrm{H}$. He, J. Klinowski, M. Forster and A. Lerf, Synthesis of graphene-based nanosheets via chemical reduction of exfoliated graphite oxide, Chem. Phys. Lett., 1998, 287(1-2), 53-56.

19 Y. W. Zhu, Graphene and graphene oxide: synthesis, properties and applications, Adv. Mater., 2010, 22, 39063924.

20 A. Abouirmrane, O. C. Compton, K. Amine and S. T. Nguyen, Non-annealed graphene paper as a binder-free anode for lithium-ion batteries, J. Phys. Chem. C, 2010, 114, 1280012804.

21 S. Stankovich, D. A. Dikin, R. D. Piner, K. A. Kohlhaas, A. Kleinhammes, Y. Jia, Y. Wu, S. T. Nguyen and R. S. Ruoff, Synthesis of graphene-based nanosheets via chemical reduction of exfoliated graphite oxide, Carbon, 2007, 45, 1558-1565.

22 S. Park, J. An, J. R. Potts, A. Velamakanni, S. Murali and R. S. Ruoff, Hydrazine-reduction of graphite- and graphene oxide, Carbon, 2011, 49, 3019-3023.

23 C. Valles, J. D. Nunez, A. M. Benito and W. K. Maser, Flexible conductive graphene paper obtained by direct and gentle annealing of graphene oxide paper, Carbon, 2012, 50, 835844.

24 C. Wang, M. D. Frogley, G. Cinque, L. Liu and A. H. Barber, Deformation and failure mechanisms in graphene oxide paper using in situ nanomechanical tensile testing, Carbon, 2013, 63, 471-477.

25 C. Wang, M. D. Frogley, G. Cinque, L. Liu and A. H. Barber, Deformation and failure mechanisms in graphene oxide paper using in situ nanomechanical tensile testing, Carbon, 2013, 63, 471-477.

$26 \mathrm{~S}$. Liu, K. Chen and Z. Bao, Reduced graphene oxide paper by supercritical ethanol treatment and its electrochemical properties, Appl. Surf. Sci., 2012, 258, 5299-5303.

27 P. Šimek, et al., "Oxygen-Free Highly Conductive Graphene Papers”, Adv. Funct. Mater., 2014, 24(31), 4878-4885.

28 D. Lamuel and G. Singh, Reduced graphene oxide paper electrode: opposing effect of thermal annealing on $\mathrm{Li}$ and Na cyclability, J. Phys. Chem. C, 2014, 118(49), 28401-28408.

29 J. Ning, J. Wang, X. Li, T. Qiu, B. Luo, L. Hao and L. Zhi, A fast room-temperature strategy for direct reduction of graphene oxide films towards flexible transparent conductive films, $J$. Mater. Chem. A, 2014, 2(28), 10969-10973.

30 M. Mauro, V. Cipolletti, M. Galimberti, P. Longo and G. Guerra, Chemically reduced graphite oxide with improved shape anisotropy, J. Phys. Chem. C, 2012, 116(46), 24809-24813.

31 L. J. Swartzendruber, Four-point probe measurement of nonuniformities in semiconductor sheet resistivity, Solid-State Electron., 1964, 7, 413.

32 L. Yan, M. Lin, C. Zeng, Z. Chen, S. Zhang, X. Zhao and M. Guo, Electroactive and biocompatible hydroxylfunctionalized graphene by ball milling, J. Mater. Chem., 2012, 22(17), 8367-8371. 
33 J. Zhou, Z. Sui, J. Zhu, P. Li, D. Chen, Y. Dai and W. Yuan, Characterization of surface oxygen complexes on carbon nanofibers by TPD, XPS and FT-IR, Carbon, 2007, 45(4), 785-796.

34 M. Mauro, M. Maggio, V. Cipolletti, M. Galimberti, P. Longo and G. Guerra, Graphite oxide intercalation compounds with rotator hexagonal order in the intercalated layers, Carbon, 2013, 61, 395-403.

35 A. C. Ferrari, Raman spectroscopy of graphene and graphite: disorder, electron-phonon coupling, doping and nonadiabatic effects, Solid State Commun., 2007, 143, 47.

36 S. Reich and C. Thomsen, Raman spectroscopy of graphite, Philos. Trans. R. Soc., A, 2004, 362, 2271.

37 M. A. Pimenta, G. Dresselhaus, M. S. Dresselhaus, L. G. Cancado, A. Jorio and R. Saito, Studying disorder in graphite-based systems by Raman spectroscopy, Phys. Chem. Chem. Phys., 2007, 9, 1276.

38 C. Castiglioni, M. Tommasini and G. Zerbi, Raman spectroscopy of polyconjugated molecules and materials: confinement effect in one and two dimensions, Philos. Trans. R. Soc., A, 2004, 362, 2425.

39 D. Graf, F. Molitor, K. Ensslin, C. Stampfer, A. Jungen, C. Hierold and L. Wirtz, Spatially resolved Raman spectroscopy of single- and few-layer graphene, Nano Lett., 2007, 7, 238.

40 C. Casiraghi, A. Hartschuh, H. Qian, S. Piscanec, C. Georgi, A. Fasoli and A. C. Ferrari, Raman Spectroscopy of Graphene Edges, Nano Lett., 2009, 9(4), 1433-1441.

41 L. R. Radovic and B. Bockrath, On the Chemical Nature of Graphene Edges: Origin of Stability and Potential for Magnetism in Carbon Materials, J. Am. Chem. Soc., 2005, 127, 5517.

42 M. Tommasini, C. Castiglioni, G. Zerbi, A. Barbon and M. Brustolon, A joint Raman and EPR spectroscopic study on ball-milled nanographites, Chem. Phys. Lett., 2011, 516(4), 220-224.

43 P. Karthika, R. Natarajan and S. D. Kaveripatnam, Functionalized exfoliated graphene oxide as supercapacitor electrodes, Soft Nanosci. Lett., 2012, 2(4), 59-66. 